\title{
Effects of Push-up Exercise with Hip Adduction on the COP Deviation and the Serratus Anterior and L1 Paraspinal Muscles
}

\author{
Min-Hee $\mathrm{Kim}^{1)}$, Won-Gyu $\mathrm{YoO}^{2 *}$ \\ 1) Institute of Health Science, Yonsei University \\ 2) Department of Physical Therapy, College of Biomedical Science and Engineering, Inje University \\ and Elderly Life Redesign Institute: 607 Obangdong, Gimhae, Gyeongsangnam-do 621-749, Republic \\ of Korea. TEL: +82 55-320-3994, FAX: +82 55-329-1678
}

\begin{abstract}
Purpose] This study investigated the effect of push-up exercise with hip adduction on the COP deviation and SA and L1 spinal muscle activation. [Subjects] Twelve males aged 20-30 years were recruited. [Methods] We measured the COP deviation and SA and L1 spinal muscle activities during push-up exercise with and without hip adduction [Results] The COP deviation significantly decreased and the SA and L1 spinal muscles were significantly increased during push-ups with hip adduction when compared with push-ups without hip adduction. [Conclusion] We thought that the push-up exercise with hip adduction might help to selectively strengthen the SA.

Key words: Thoracolumbar fascia, Push up exercise, Serratus anterior
\end{abstract}

(This article was submitted Jan. 15, 2013, and was accepted Mar. 1, 2013)

\section{INTRODUCTION}

A push-up is a common closed kinetic chain exercise for the upper extremity that is known to activate the scapulothoracic musculature ${ }^{1)}$. A closed kinetic chain exercise could facilitate the stability of an articular joint through muscle co-activation, which would then lead to enhanced dynamic stabilization of the shoulder girdle in patients with shoulder disorders ${ }^{1,2)}$. Thomas ${ }^{3)}$ reported that the hip adductor muscle is connected to the internal abdominal muscle through the iliacus, psoas major, and quadratus lumborum. Kim and $\mathrm{Yoo}^{4)}$ reported that the muscle activities of the external oblique, internal oblique, and L5 paraspinal muscles increased during hip adduction using a visual feedback device. Maenhout et al. ${ }^{5)}$ reported scapular EMG activity during knee push-up-plus (KPP) and six commonly used variations. When using a kinetic-chain approach during KPP, homolateral leg extension increased SA activity because the stress of the thoracolumbar fascia was transmitted to the heterolateral scapula ${ }^{5)}$. However, few studies have investigated the effect of hip adduction on shoulder muscle activation during a push-up exercise. So, we investigated the effect of push-up exercise with hip adduction on the COP deviation and SA and L1 spinal muscle activation.

\section{SUBJECTS AND METHODS}

Twelve males aged 20-30 years and with a mean height and weight of $174.7 \pm 5.0 \mathrm{~cm}$ and $68.5 \pm 6.4 \mathrm{~kg}$, respectively, participated in this study. The subjects had no his-

*To whom correspondence should be addressed. E-mail: won7y@inje.ac.kr tory of musculoskeletal disorders or pain associated with the upper and lower extremities in the past 6 months. All participants gave their informed, written consent according to the protocol approved by Inje University Faculty of Health Science Human Ethics Committee. The variance of the COP between hands was measured using a Tekscan system (Tekscan Inc., South Boston, MA, USA) during a push-up progression. The changes in COP were represented as values of standard deviation ${ }^{6}$. The pressure sensor signals were sampled at $50 \mathrm{~Hz}$. All EMG signals were amplified, band-pass filtered $(20-500 \mathrm{~Hz})$, and then sampled at $1,000 \mathrm{~Hz}$ using the Acqknowledge 3.9.1 software. The amplitude was normalized by the maximal voluntary isometric contraction. We measured the serratus anterior (SA) and L1 level paraspinal (L1 spinal) muscles. A digital air-pressure sensor (AP Series Pressure Sensor, Keyence, Osaka, Japan) was directly connected to an air cushion and calibrated to the zero point during a no hip adduction exercise. We designed the air cushion to exert a continuous external load using the tension air cushion against the medial side of the thigh. All trials were started in the standardized push-up starting position with the hands one shoulder width apart and the participant's middle finger under the acromioclavicular joint, as described in a previous study. The COP was calibrated to the zero point in the standardized position. Participants performed 2 different trials: a push-up exercise with and without hip adduction. The trials were presented in a randomized order, and each participant was allowed to rest for 3 minutes between each trial. The Statistical Package for the Social Sciences (SPSS, Chicago, IL, USA) was used to perform the paired t-test to analyze the differences in COP deviation and SA and L1 spinal muscle activities during push-up exercise with and without hip adduction. 
The alpha level for statistical significance was set at 0.05 .

\section{RESULTS}

The COP deviation was significantly decreased during push-ups with hip adduction $(5.6 \pm 2.0 \mathrm{~mm})$ when compared with push-ups without hip adduction $(7.5 \pm 2.7 \mathrm{~mm})$. The activity of the SA was significantly increased during pushups with hip adduction $(52.0 \pm 10.6 \%)$ when compared with push-ups without hip adduction $(48.3 \pm 12.9 \%)$. The L1 spinal activity was significantly increased during push-ups with hip adduction $(36.5 \pm 9.1 \%)$ when compared with pushups without hip adduction $(28.9 \pm 15.7 \%)$.

\section{DISCUSSION}

This study investigated the effect of a push-up exercise with hip adduction on the COP deviation and SA and L1 spinal muscle activation. Park et al. ${ }^{6}$ reported a negative correlation between serratus anterior activation and displacement of the COP. The COP deviation was significantly decreased during push-ups with hip adduction when compared with push-ups performed without hip adduction. In the present study, the SA showed greater activation with low COP deviation during a push-up with hip adduction. The hip adductor muscle contraction synergistically facilitates contractions of the pelvic floor and abdominal muscles $^{7)}$. First, this co-activation of the hip adductor muscle, pelvic floor muscle, and internal abdominal muscle is necessary for the induction of intra-abdominal pressure, and it powerfully reinforces the multifidus muscles and contributes to spinal stability ${ }^{8}$. We thought that a push-up exercise with hip adduction would increase the trunk stability. Second, a previous study also showed that homolateral leg extension increased SA activity because the stress of the thoracolumbar fascia was transmitted to the heterolateral scapula $^{5)}$. Some back paraspinals attach to the thoracolumbar fascia. The muscles that originate from the thoracolumbar fascia, and that are superficially located, play a major role in transmitting the load of the trunk to the arms and shoulders $^{3,9)}$. In the present study, the L1 spinal muscle activity was significantly increased during a push-up with hip adduction. Our opinion was that a push-up exercise with hip adduction increased the trunk stability by co-activating the thoracolumbar fascia. Finally, a push-up exercise with hip adduction increased the SA activity because the stress on the thoracolumbar fascia was transmitted to the scapula.

\section{ACKNOWLEDGEMENT}

This research was supported by Basic Science Research Program through the National Research Foundation of Korea (NRF) funded by the Ministry of Education, Science and Technology (No. 2012R1A1B4001058).

\section{REFERENCES}

1) Tucker WS, Armstrong CW, Gribble PA, et al.: Scapular muscle activity in overhead athletes with symptoms of secondary shoulder impingement during closed chain exercises. Arch Phys Med Rehabil, 2010, 91: 550-556. [Medline] [CrossRef]

2) Ellenbecker TS, Cappel K: Clinical applications of closed kinetic chain exercises in the upper extremities. Orthop Phys Ther Clin N Am, 2000, 9 : 231-245.

3) Thomas WM: Anatomical Trains; Myofascial Meridians for Manual and Movement Therapists, 2nd ed. London: Churchill Livingstone, 2009.

4) Kim MH, Yoo WG: Effects of a visual feedback device for hip adduction on trunk muscles and sitting posture in visual display terminal workers. Asia Pac J Public Health, 2011, 23: 378-385. [Medline] [CrossRef]

5) Maenhout A, Praet KV, Pizzi L, et al.: Electromyographic analysis of knee push up plus variations: what's the influence of the kinetic chain on scapular muscle activity? Br J Sports Med, 2010, 44: 1010-1015. [Medline] [CrossRef]

6) Se-yeon P, Won-gyu Y, Kwon H, et al.: Scapulothoracic muscle activation on stable and unstable support surfaces. Int J Athl Ther Trai, 2013, 18: $25-29$.

7) Bø K, Stien R: Needle EMG registration of striated urethral wall and pelvic floor muscle activity patterns during cough, Valsalva, abdominal, hip adductor, and gluteal muscle contractions in nulliparous healthy females. Neurourol Urodyn, 1994, 13: 35-41. [Medline] [CrossRef]

8) Hemborg B, Moritz U, Hamberg J, et al.: Intraabdominal pressure and trunk muscle activity during lifting-effect of abdominal muscle training in healthy subjects. Scand J Rehabil Med, 1983, 15: 183-196. [Medline]

9) Neumann DA: Kinesiology of the musculoskeletal system: foundations for physical rehabilitation. St Louis: Mosby, 2002. 